\title{
The differential protein and lipid compositions of noncaveolar lipid microdomains and caveolae
}

\author{
Yao Yao ${ }^{1}$, Shangyu Hong ${ }^{1}, \mathrm{Hu}$ Zhou$^{2}$, Taichang Yuan ${ }^{1}$, Rong Zeng ${ }^{2}$, Kan Liao ${ }^{1}$

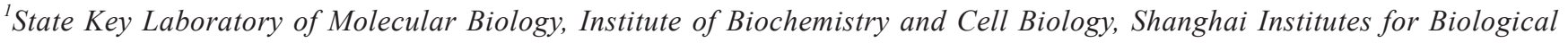 \\ Sciences, Chinese Academy of Sciences, Shanghai 200031, China; ${ }^{2}$ Research Center for Proteome Analysis, Institute of Biochemis- \\ try and Cell Biology, Shanghai Institutes for Biological Sciences, Chinese Academy of Sciences, Shanghai 200031, China
}

Morphologically, caveolae and lipid rafts are two different membrane structures. They are often reported to share similar lipid and protein compositions, and are considered to be two subtypes of membrane lipid microdomains. By modifying sucrose density gradient flotation centrifugation, which is used to isolate lipid microdomains, we were able to separate caveolae and noncaveolar lipid microdomains into two distinct fractions. The caveolar membranes are membrane vesicles of 100-nm diameter, enriched with caveolin-1 and flotillin-1. The noncaveolar lipid microdomains are amorphous membranes and most likely the coalescence of heterogeneous lipid rafts. They are depleted of caveolin-1 and are more enriched with cholesterol and sphingolipids than the caveolae. Many membrane proteins, such as insulin-like growth factor-1 receptor (membrane receptor), aquaporin-1 (membrane transporter), Thy-1 and Ncadherin (glycosylphosphatidylinositol-anchored membrane protein and membrane glycoprotein), are specifically associated with noncaveolar lipid microdomains, but not with caveolae. These results indicate that the lipid and protein compositions of caveolae differ from those of noncaveolar lipid microdomains. The difference in their protein compositions implies that these two membrane microdomains may have different cellular functions.

Keywords: membrane lipid microdomains, caveolae, noncaveolar lipid microdomains, lipid rafts, sucrose density gradient, sodium carbonate extraction

Cell Research (2009) 19:497-506. doi: 10.1038/cr.2009.27; published online 3 March 2009

\section{Introduction}

Caveolae have been widely accepted as distinct plasma membrane structures $[1,2]$. The formation of 50 - to 100 $\mathrm{nm}$ invaginations on the plasma membrane is associated with the presence of the caveolin-1 protein, and caveolae cannot form in the absence of caveolin-1 [3-5]. Besides their morphological visibility on the plasma membrane [2], caveolar membranes are biochemically defined by (a) resistance to solubilization by Triton $\mathrm{X}-100$ at $4{ }^{\circ} \mathrm{C}$, (b) a light, buoyant density and (c) enrichment with sphingolipids and cholesterol [2]. However, membrane fractions with these biochemical characteristics can be isolated in cells

Correspondence: Kan Liao

Tel: +86-21-54921113; Fax: +86-21-54921011

E-mail:kliao@sibs.ac.cn

Abbreviation: GPI-APs (glycosylphosphatidylinositol-anchored proteins) Received 11 June 2008; revised 5 September 2008; accepted 25 September 2008; published online 3 March 2009 without caveolins and caveolae [2, 5-8]. For this reason, broader terms, such as detergent-resistant membranes or membrane lipid microdomains, are used to define these membrane fractions.

In contrast to caveolae that are readily identifiable membrane invaginations marked by caveolin-1, lipid rafts cannot be easily distinguished from the surrounding membrane [6-8]. The dynamic nature of lipid rafts makes it impossible to physically define a specified set of membranes as lipid rafts. Lipid rafts are now considered a spatiotemporally regulated dynamic lipid-dependent segregation of specific membrane components [9-14]. They range from unstable nanoscale clusters of proteins, cholesterol and sphingolipids, to relatively stable microdomains formed from preexisting small clusters through protein-protein or protein-lipid interactions. Thus, any isolated noncaveolar lipid microdomains are essentially an equilibrated mixture of heterogeneous dynamic membrane lipid rafts.

Biochemically, both caveolae and lipid rafts exhibit a 
resistance to detergent extraction and are co-isolated by light-density flotation centrifugation $[1,2,5,8]$. Characterizing this kind of membrane lipid microdomains reflects the mixed properties of caveolae and lipid rafts. For the biochemical analysis of caveolae or lipid rafts, it is essential to separate caveolae and lipid rafts from each other. It may be impossible to isolate a defined set of lipid rafts for characterization, but the well-defined caveolae can at least be isolated from the rest of the heterogeneous lipid rafts.

In our previous study with caveolin-1 knockdown 3T3-L1 cells, we found that suppressing caveolin-1 through RNA interference ablated the caveolae [5]. However, the fractionation of membrane lipid microdomains and the association of the insulin-like growth factor-1 (IGF-1) receptor with lipid microdomains are not affected by the ablation of caveolae, nor is IGF-1 receptor signaling affected during 3T3-L1 adipocyte differentiation induction [5]. Although IGF-1 receptor signaling is sensitive to cholesterol depletion, it is independent of caveolae and caveolin-1 [5]. In the differentiated caveolin-1 knockdown 3T3-L1 adipocyte, the absence of caveolae has no adverse effect on insulin receptor signaling, GLUT4 translocation or glucose uptake [15]. These results indicate that IGF-1 receptor and insulin receptor signaling are membrane cholesterol sensitive, but caveolae independent.

In the present study, we separated caveolin-1-enriched caveolar membranes (caveolae) from the heterogeneous noncaveolar lipid microdomains in 3T3-L1 cells by a modified four-step sucrose density gradient. Cholesterol and sphingolipids were more enriched in noncaveolar lipid microdomains than in caveolae. The specific association of IGF-1 receptor, Thy-1, c-Yes and aquaporin-1 with noncaveolar lipid microdomains suggested that the dynamic lipid rafts might be the functionally important membrane platform for receptor transmembrane signaling.

\section{Results}

Detergent and non-detergent extraction of membrane lipid microdomains from isolated plasma membranes

3T3-L1 cells have a high abundance of both caveolae and lipid rafts [5]. Because membrane lipid microdomains (caveolae and lipid rafts) are mostly located on the plasma membrane $[2,8]$, purified 3T3-L1 cell plasma membranes were used to extract membrane lipid microdomains. Plasma membrane purity was examined by membrane markers and electron microscopy (Figure 1A1C). In the purified plasma membrane fraction, which was marked by $\mathrm{N}$-cadherin and caveolin-1, there was no endoplasmic reticulum (calnexin), mitochondria (cyto- chrome $c$ ) or nuclei (histone H1) (Figure 1B). The lysosome (cathepsin D) and Golgi ( $\gamma$-adaptin) membranes were dissolved by sodium carbonate extraction and were not present in the fractionated insoluble lipid microdomain fractions (Figure 1E).

Two extraction methods are often used to isolate membrane lipid microdomains: low-concentration Triton X-100 solution or detergent-free, $\mathrm{pH} 11$, sodium carbonate solution [16-18]. In combination with sucrose density gradient flotation centrifugation, the insoluble membrane lipid microdomains can be isolated by flotation in a sucrose density gradient. Triton X-100 extraction completely dissolved the membrane lipid microdomains from the isolated plasma membrane (Figure 1D). The lipid microdomain-associated proteins caveolin-1, flotillin-1 [19] and IGF-1 receptor [5, 20] were all dissolved by the Triton X-100 solution (Figure 1D). In contrast, the sodium carbonate solution could extract lipid microdomains from the purified plasma membrane (Figure 1D). Caveolin-1, flotillin-1 and IGF-1 receptor were all associated with low-density sphingolipid-enriched insoluble membranes (Figure 1D). Because Triton X-100 extraction was affected by the amount of cellular membrane mass (Supplementary information, Figures S1 and S2), detergent-free sodium carbonate was used in subsequent studies with purified plasma membrane.

\section{Separation of caveolae and noncaveolar lipid microdo- mains}

Caveolae are stable membrane structures that are well defined by their invaginated morphology and the presence of the marker protein, caveolin-1 $[1,2]$. The dynamic lipid rafts are composed of unstable nanoclusters and stabilized coalescences of nanoclusters in the plasma membrane $[10,11]$. These two groups of membrane microdomains were floated as a single peak of cholesteroland sphingolipid-enriched membrane microdomains at the interface of $5-35 \%$ sucrose in the conventional threestep sucrose density gradient (Figure 2A and 2B). Cholesterol, gangliosides (glycosphingolipids) and associated proteins (caveolin-1, flotillin-1 and IGF-1 receptor) were co-isolated in low-density insoluble membrane fractions (Figures 1D and 2B).

In caveolin-1 knockdown 3T3-L1 cells in which caveolae are absent, the IGF-1 receptor is still associated with membrane lipid microdomains [5]. In addition, the insulin receptor is associated with membrane microdomains enriched with gangliosides, but devoid of caveolin-1 [15]. These findings suggest that a ganglioside-enriched lipid microdomain is separable from caveolae. By inserting an intermediate sucrose density step between the $5 \%$ and $35 \%$ sucrose steps of the conventional $5-35-45 \%$ sucrose 
A

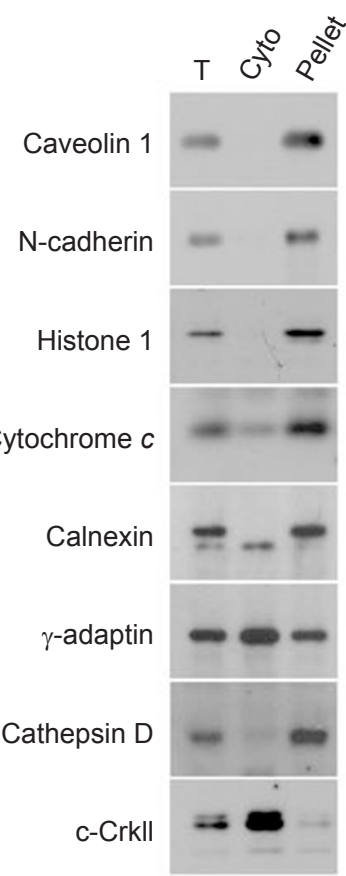

$B$

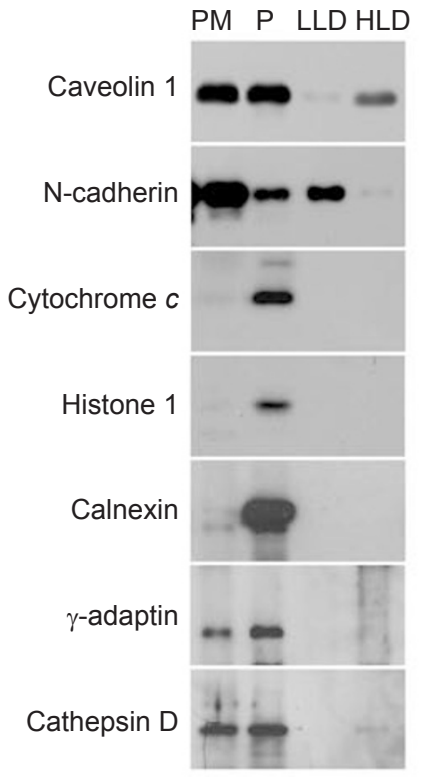

C

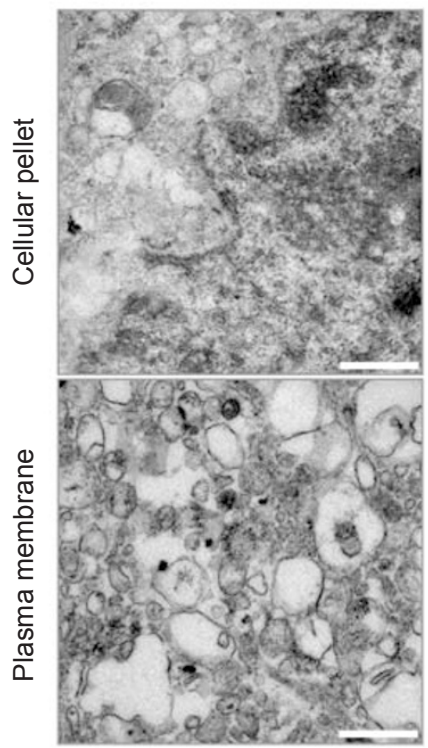

D

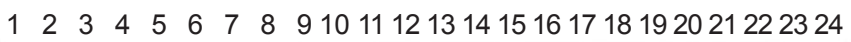

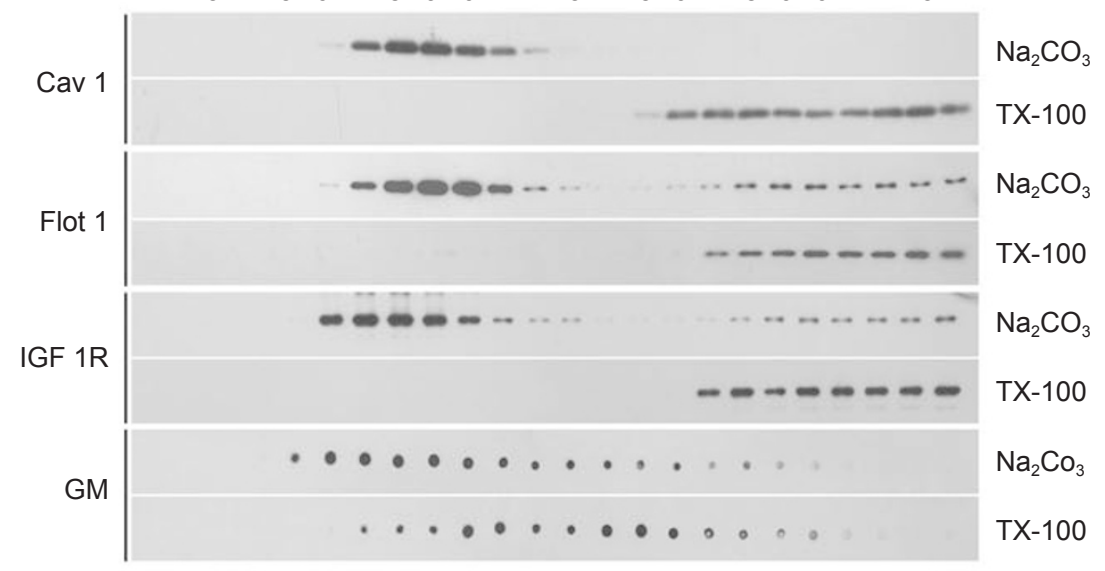

E

$1223 \quad 45567889101112131415161718192021222324$

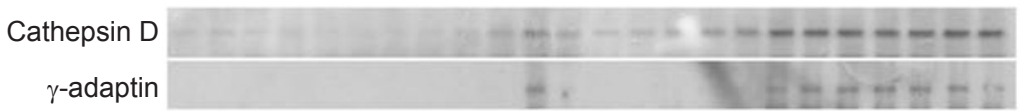

Figure 1 Purification of the plasma membrane and extraction of membrane lipid microdomains. (A) Separation of cellular membranes from cytoplasm. T indicates the whole cell lysate. Cyto indicates the supernatant of $10000 \times g$ centrifugation. Pellet indicates the pellet of $10000 \times g$ centrifugation and was used to purify the plasma membrane. Proteins were detected by western blot. (B) Purification of the plasma membrane. PM indicates the plasma membrane collected at the interface of the sucrose cushion, and $\mathrm{P}$ indicates the pellet at the bottom of the sucrose cushion. LLD and HLD, respectively, indicate the light low-density noncaveolar lipid microdomains and the heavy low-density caveolae, as described in Figure 2. (C) Electron microscopic view of the purified plasma membrane. Plasma membrane at the interface of the sucrose cushion (plasma membrane) and cellular organelles sedimented at the bottom of the sucrose cushion (cellular pellet) were examined by electron microscopy. The scale bar is $500 \mathrm{~nm}$. (D) Membrane lipid microdomains extracted by detergent or non-detergent solutions from the purified plasma membrane. Plasma membrane (containing $80 \mu \mathrm{g}$ protein) was extracted with detergent (TX-100) or non-detergent $\left(\mathrm{Na}_{2} \mathrm{CO}_{3}\right)$ solution and fractionated with a three-step sucrose gradient. Caveolin-1 (Cav 1), flotillin-1 (Flot 1) and IGF-1 receptor (IGF 1R) were detected by western blot. Gangliosides (GM) were dot blotted with the horseradish peroxidase-labeled cholera toxin $\beta$-subunit. (E) Golgi and lysosome marker proteins in membrane microdomains extracted with sodium carbonate solution and separated with sucrose density gradient centrifugation. CTS D, cathepsin D; $\gamma$-ADT, $\gamma$-adaptin. 
A

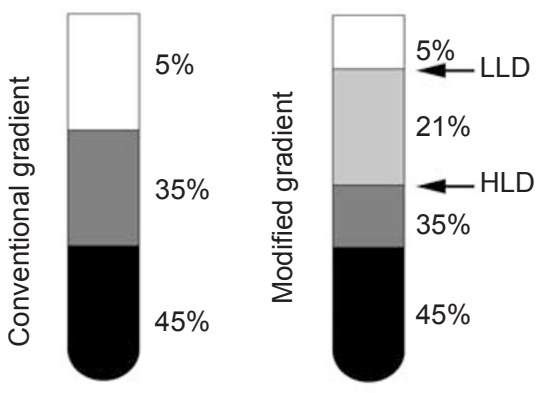

B

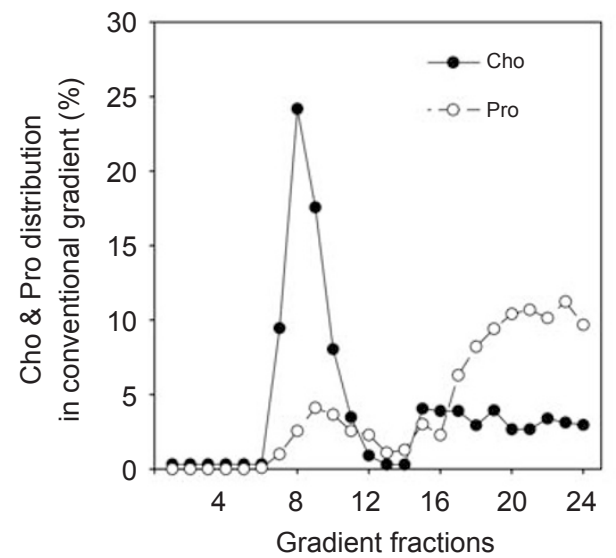

C

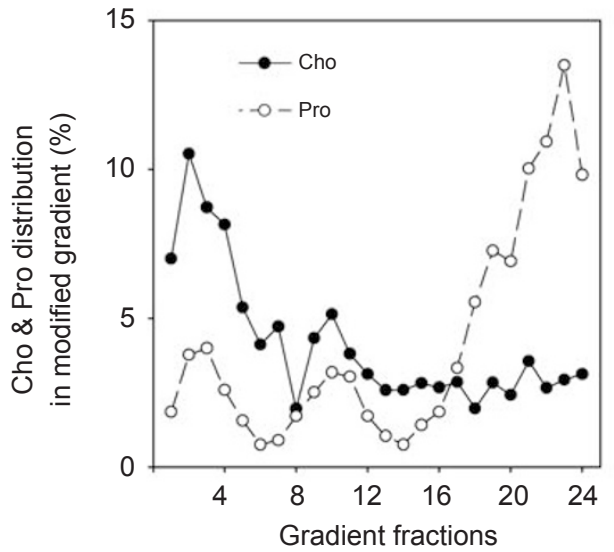

D $1 \quad 2 \quad 3 \quad 4 \quad 5 \quad 6 \quad 7 \quad 8 \quad 9101112131415161718192021222324$

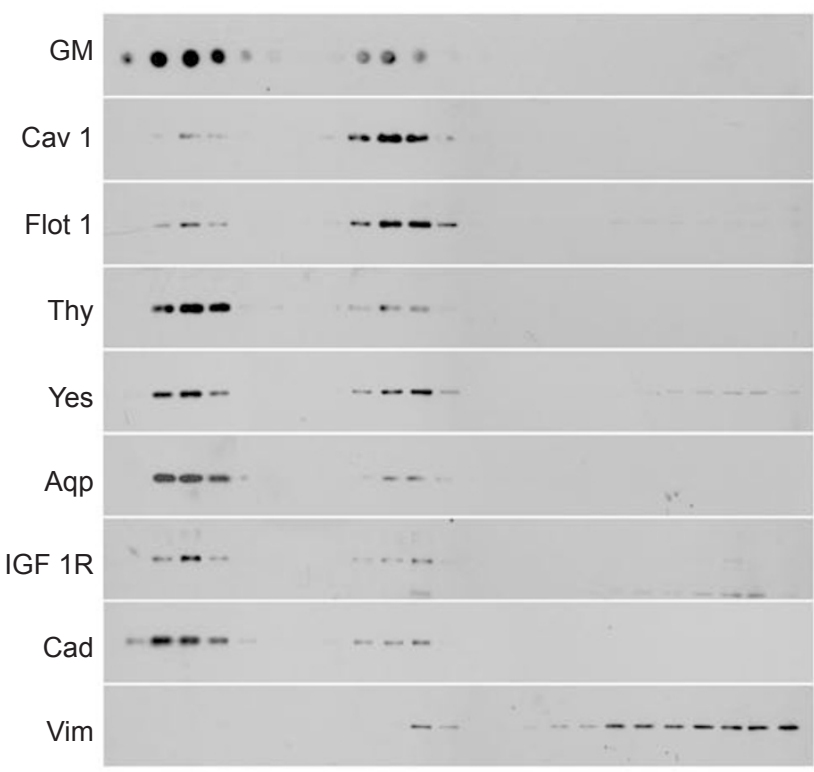

$E$

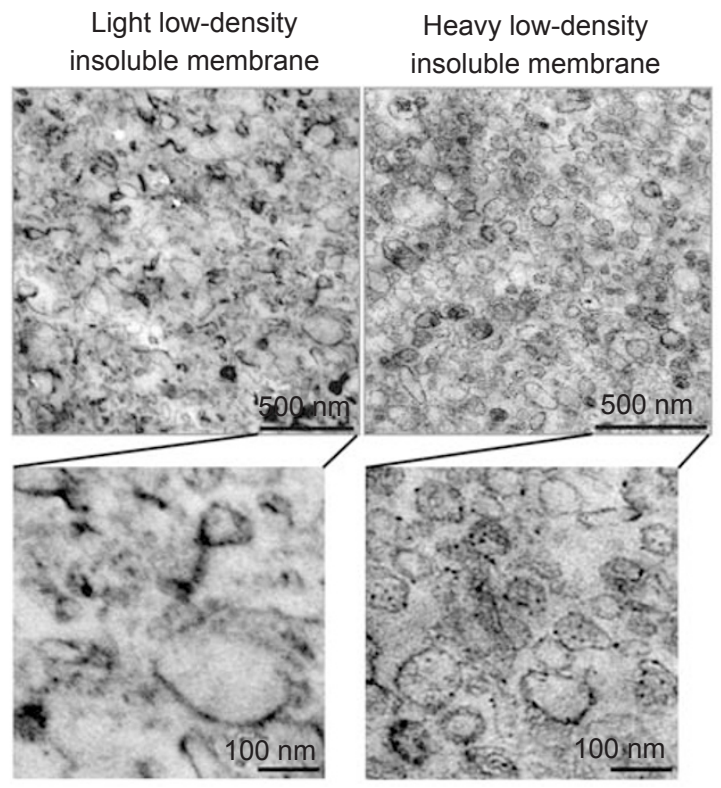

Figure 2 Separation of caveolae and noncaveolar lipid microdomains from the purified plasma membrane. (A) Illustration of a sucrose density gradient. The percentages indicate the sucrose concentration (w/v). LLD and HLD indicate the light low-density and heavy low-density interfaces, respectively. (B) Cholesterol and protein in the fractions of the conventional three-step sucrose density gradient for sodium carbonate-extracted plasma membranes. Membrane microdomains were extracted and fractionated from purified plasma membrane $(200 \mu \mathrm{g}$ protein). The amounts of cholesterol (Cho) and protein (Pro) in each fraction were determined. The results are from one representative experiment. (C) Cholesterol and protein in the fractions of the modified four-step sucrose density gradient for sodium carbonate-extracted plasma membranes. The labels are the same as in panel (B). The results are from one representative experiment. (D) Analysis of lipid microdomain-associated proteins and gangliosides in the fractions of the modified four-step sucrose gradient. Gangliosides (GM) are major lipids in lipid microdomains. Caveolin-1 (Cav 1) is the marker protein of caveolae. Flotillin-1 (Flot 1) is a marker for a lipid microdomain structure protein. Thy-1 (Thy) is a GPI-anchored protein. c-Yes (Yes) is an inner-membrane surface lipid-tethered protein. Aquaporin-1 (Aqp) is an integrated membrane transporter. IGF-1 receptor (IGF 1R) is a membrane receptor. N-cadherin (Cad) is a surface glycoprotein. Vimentin ( $\mathrm{Vim})$ is the cytoskeleton-associated protein that is also reported to be caveolae associated [26]. (E) Electron microscopic view of isolated light low-density membrane lipid microdomains and heavy low-density membrane lipid microdomains. 
density gradient, we modified the conventional threestep sucrose density gradient into a four-step density gradient (Figure 2A) [15]. A 21\% sucrose step achieved the best separation of caveolin-1-associated membranes from IGF-1 receptor-associated and ganglioside-enriched membranes (Figure 2C and 2D). In this modified fourstep sucrose density gradient, the membrane lipid microdomains were fractionated into two interfaces of 5-21\% sucrose and 21-35\% sucrose (Figure 2C). The two-membrane lipid microdomain fractions had similar amounts of proteins, but different amounts of cholesterol (Figure $2 \mathrm{C}$ ). The insoluble membrane lipid microdomains at the $5-21 \%$ interface contained higher levels of cholesterol and sphingolipids, which caused their lighter density (Figure 2C and 2D).

To identify the caveolae, these two membrane fractions were subjected to western blot for caveolin-1. As shown in Figure 2D, caveolin-1 was mostly fractionated into the heavy low-density lipid microdomains (at the $21-35 \%$ sucrose interface). The caveolin-1-enriched membrane microdomains were also visualized by elec- tron microscopy. They appeared as membrane vesicles with a diameter of around $100 \mathrm{~nm}$ that resemble the morphology of caveolae (Figure 2E) [2].

The light low-density lipid microdomains (at the $5-21 \%$ sucrose interface) were devoid of caveolin-1 (Figure 2D). They were amorphous membranes, indicating a heterogeneous nature (Figure 2E). The membrane heterogeneity, the enrichment of cholesterol and sphingolipids, and the absence of caveolin-1 all suggested that the light low-density noncaveolar lipid microdomains are a heterogeneous coalescence of lipid rafts (Figure 2C and 2D) [6,9-11]. The modified sucrose density gradient clearly separated two different types of membrane microdomains.

After caveolae are separated from the other noncaveolar lipid microdomains (most likely the coalescence of heterogeneous lipid rafts), it is possible to analyze their associated proteins. Many lipid microdomain-associated proteins, such as Thy-1 (GPI-AP, glycosylphosphatidylinositol-anchored protein) [21], N-cadherin (membrane glycoprotein) $[22,23]$, aquaporin-1 (membrane
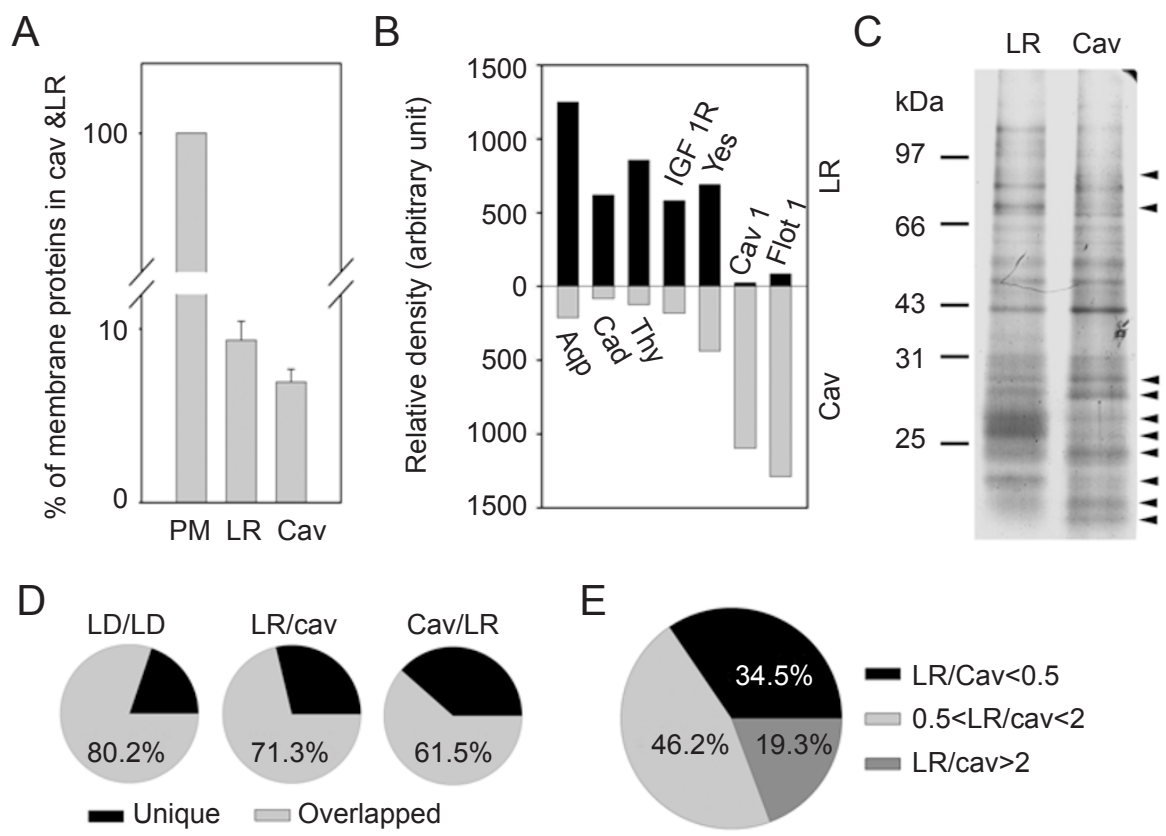

Figure 3 Protein profiling in caveolae and noncaveolar lipid microdomains. (A) The amounts of protein in noncaveolar lipid microdomains and caveolae. The amounts of protein in plasma membrane (PM), noncaveolar lipid microdomains (LR) and caveolae (cav) was determined. The results are the average of three experiments. (B) Densitometric quantification of proteins identified by western blot. The results are from one representative experiment. (C) SDS-PAGE of proteins in noncaveolar lipid microdomains and caveolae. The proteins were revealed by silver staining. (D) Protein profiling by shot-gun mass spectrometry analysis. LD/LD, protein comparison of two lipid microdomain samples isolated by the conventional sucrose gradient; LR/ cav, comparison of proteins identified in noncaveolar lipid microdomains with those in caveolae; cav/LR, comparison of proteins identified in caveolae with those in noncaveolar lipid microdomains. (E) Label-free quantification for overlapped proteins. $\mathrm{LR} / \mathrm{cav}<0.5,0.5<\mathrm{LR} / \mathrm{cav}<2$, and LR/cav $>2$ indicate the ratios of the protein amounts in noncaveolar lipid microdomains to those in caveolae. 
transporter) [24, 25], IGF-1 receptor (membrane tyrosine kinase receptor) $[5,20]$ and c-Yes (inner-membrane lipid-tethered protein) [18], were actually associated with noncaveolar lipid microdomains (Figure 2D). Flotillin-1, a structural protein in membrane lipid microdomains [19], was associated with caveolae (Figure 2D). Although vimentin is reported to associate with lipid microdomains [26], only a small fraction of the protein was detected in the caveolar membrane fractions (Figure 2D). These results suggest that proteins are specifically associated with caveolae or noncaveolar lipid microdomains.

Lipid and protein compositions in caveolae and noncaveolar lipid rafts

Most proteins in the purified plasma membrane were not associated with lipid microdomains, which contained $16 \%$ of all membrane proteins ( $7 \%$ in caveolae and $9 \%$ in noncaveolar lipid microdomains; Figure 3A). Most lipid microdomain-associated proteins exhibited specificity for caveolae or noncaveolar lipid microdomains (Figures 2D and 3B). The SDS-polyacrylamide gel electrophoresis (PAGE) of caveolae and noncaveolar lipid microdomains proteins also showed different protein profiles (Figure 3C). For a better comparison of the proteins associated with caveolae and noncaveolar lipid microdomains, shot-gun mass spectrometry was used to analyze proteins in caveolae and in noncaveolar lipid microdomains. A total of $61.5 \%$ of the proteins identified in caveolae overlapped with the proteins in noncaveolar lipid microdomains, and $71.3 \%$ of the proteins identified in noncaveolar lipid microdomains overlapped with the caveolar proteins (Figure 3D). For two identical lipid microdomain samples, however, $80.2 \%$ of the proteins identified by mass spectrometry overlapped (Figure 3D). The low overlap rate between caveolae and noncaveolar lipid microdomains suggested different protein profiles. Because of the high sensitivity of mass spectrometry analysis, this overlap did not imply an equal amount of protein in the two samples. A label-free comparison method was used to quantify differences between the overlapped proteins [27]. As shown in Figure 3E, more than half of the overlapped proteins had over a two-fold expression difference between caveolae and noncaveolar lipid microdomains. Taken together, these results suggest that the caveolae and noncaveolar lipid microdomains separated by the modified sucrose density gradient had different protein compositions.

The most important characteristic of caveolae and lipid rafts is the enrichment of cholesterol and sphingolipids [2, 6-8]. Our results indicated that lipid rafts were far more enriched with cholesterol and gangliosides (Figure $2 \mathrm{C}$ and $2 \mathrm{D}$ ). The use of thin-layer chromatography analysis [28-30] demonstrated that noncaveolar lipid microdomains contained more sphingomyelin, phosphatidylcholine and phosphatidylethanolamine (Figure 4A). In comparison to caveolae, noncaveolar lipid microdomains contained twice the amount of cholesterol per microgram of proteins (Figure 4B). The total amount of protein in noncaveolar lipid microdomains was only 1.2 -fold of that in caveolae (Figures $2 \mathrm{C}$ and $3 \mathrm{~A}$ ). However, the total amounts of cholesterol, gangliosides, sphingomyelin, phosphatidylcholine and phosphatidylethanolamine were
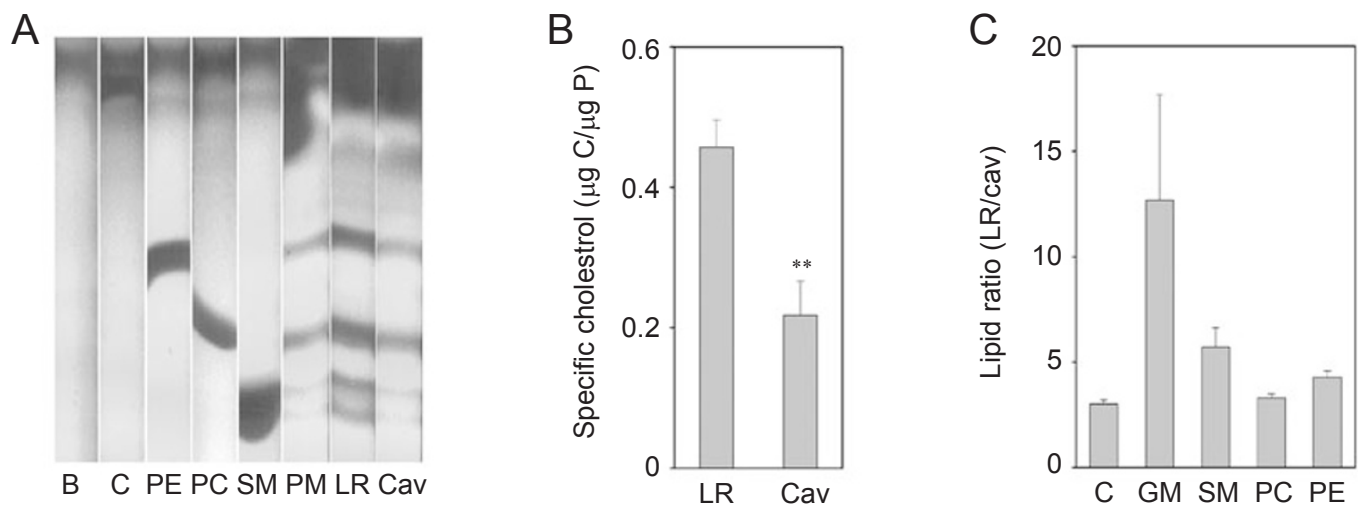

Figure 4 Analysis of lipids in caveolae and noncaveolar lipid microdomains. (A) Thin-layer chromatography of lipids extracted from the plasma membrane (PM), light low-density noncaveolar lipid microdomains (LR) and heavy low-density caveolae (cav). The samples were normalized to the equivalent of $20 \mu \mathrm{g}$ membrane protein. $\mathrm{B}$ is a solvent blank. Five micrograms of standard of cholesterol (C), phosphatidylethanolamine (PE), phosphatidylcholine (PC) and sphingomyelin (SM) are used as standards. (B) Measurement of specific cholesterol in noncaveolar lipid microdomains (LR) and caveolae (cav). The cholesterol is presented as micrograms of cholesterol per microgram of protein ( $\mu \mathrm{g} \mathrm{C} / \mu \mathrm{g} \mathrm{P}$ ). The results are the average of six experiments. ${ }^{* *} P<0.001$. (C) The ratio of total lipids in noncaveolar lipid microdomains to those in caveolae. 
several folds higher in noncaveolar lipid microdomains (Figure 4C). Thus, noncaveolar lipid microdomains were much more lipid enriched than caveolae.

\section{Discussion}

Without a definite morphology, it is difficult to describe what types of lipid rafts are present in a living cell. It was originally proposed that "lateral organization probably results from preferential packing of sphingolipids and cholesterol into moving platforms, or rafts, onto which specific proteins attach within the bilayer" [6]. However, recent studies show that stable, preexisting lipid rafts do not exist in live cell membranes [11, $13,31]$. Instead, they exist in a state of nanoclusters and monomers in a dynamic balance $[13,32]$. As small nanoclusters collide with each other and coalesce to form larger lipid-based assemblies, the existence of stable microdomains at $\geq 1000 \mathrm{~nm}$ can be identified [9, 14, 33]. In our current study, the "noncaveolar lipid microdomains" separated from caveolae may be a mixture of heterogeneous rafts ranging from nanoclusters to stable microdomains. Morphologically, they are a mixture of an amorphous substance with heterogeneous membrane pieces (Figure 2E). Biochemically, they contain associated GPI-APs and are enriched with cholesterol, gangliosides and sphingomyelin (Figures 2D and 4).

Compared with the stable caveolae, the short-lived unstable nanoscale clusters are more efficient for signal transduction [34-36]. Each nanocluster produces a signal in a way of quantized burst, so the cell can easily modulate the timing and amplitude of the signal simply by synchronizing the quantity of these nanoclusters [34, 35]. Our results with caveolin-1 knockdown 3T3-L1 cells indicate that lipid rafts are the signal platform for the IGF1 receptor [5]. In the current study, molecules involved in signal transduction are mostly enriched in noncaveolar lipid microdomains, but not in caveolae (Figure 2D). Apart from the proteins shown in Figure 2D, many other signal molecules (e.g., EGF receptor, G protein family and JAK1 (identified by mass spectrometry analysis)) are also enriched in noncaveolar lipid microdomains. However, the caveolar fraction is more enriched with molecules related to the cytoskeletal system, such as dynamin-2, filamin $\mathrm{B}$ and $\alpha$-actinin (identified by mass spectrometry analysis). The function of caveolae in GLUT4 internalization is in good agreement with these identified caveolar proteins [15].

Unlike the heterogeneous lipid rafts, the isolated caveolae are rather "morphologically homogeneous" (Figure $2 \mathrm{E}$ ). The separation of caveolae from noncaveolar lipid microdomains resolves the ambiguity caused by analyzing the lipid microdomains that are isolated with the conventional sucrose density gradient. For example, T-cadherin is reported as a caveolae-associated protein in one study and as a lipid raft-associated protein in another study [37, 38]. Aquaporin-1, Thy-1, IGF-1 receptor and $\mathrm{N}$-cadherin, which are reported to co-fractionate with caveolin-1 [5, 20, 21-25], are actually noncaveolar lipid microdomain-associated proteins (Figure 2D). Thus, this modified sucrose density gradient provides a better technique with which to study caveolae.

Cholesterol depletion by methylcyclodextrin or filipin is often used to study the cellular function of caveolae and lipid rafts [5, 27, 39-42]. Because cholesterol is present in both caveolae and lipid rafts (Figure 4), both will be disrupted by cholesterol depletion. Thus, the cellular effect of cholesterol depletion cannot differentiate between the functions of caveolae and lipid rafts. Although caveolae have been reported to be important for insulin receptor signaling and glucose uptake in studies using the cholesterol depletion method [39-42], specifically targeting caveolae by a caveolin-1 knockdown indicates caveolae-independent insulin receptor signaling and glucose uptake $[5,15]$. By separating lipid rafts from caveolae, noncaveolar lipid microdomain-associated insulin receptor and GLUT4 are observed [15].

In the published literature, both caveolae and lipid rafts are often considered to be cholesterol- and sphingolipid-enriched membrane lipid microdomains $[2,8$, 43]. However, the lipid contents in these two lipid microdomains are quite different (Figure 4). Caveolae are not as lipid-enriched as lipid rafts. In fact, sphingolipidenriched, but caveolin-depleted, membrane microdomains can be isolated from mouse melanoma B16 cells by immunoprecipitation with an anti-GM3 ganglioside antibody [44]. Caveolae are also different membrane fractions from sphingolipid-enriched membrane microdomains in MDCK II cells [45]. These reports and our results suggest that caveolae and lipid rafts are quite different membrane microdomains and that they should be analyzed separately.

\section{Materials and Methods}

\section{Materials}

Anti-aquaporin-1, anti-caveolin-1 $\alpha$-isoform, anti-IGF-1 receptor $\beta$-subunit, anti-vimentin, anti-cytochrome $c$, anti-histone $\mathrm{H} 1$, anti-calnexin, anti- $\gamma$-adaptin and anti-cathepsin D antibodies were purchased from Santa Cruz Biotechnology, Inc. (Santa Cruz, CA, USA). Anti-N-cadherin, anti-c-Yes, anti-flotillin-1 and anti-c-Crk antibodies were obtained from BD Transduction Laboratories (San Jose, CA, USA). Anti-Thy-1 antibody, horseradish peroxidase (HRP)-conjugated cholera toxin $\beta$-subunit, HRP-conjugated secondary antibodies, phosphatidyl choline, phosphatidyl ethanol- 
amine and sphingomyelin were purchased from Sigma (St Louis, MO, USA).

\section{Extraction of membrane lipid microdomains and sucrose} density gradient flotation centrifugation

3T3-L1 cells were cultured in Dulbecco's modified Eagle's medium (DMEM) with 10\% calf serum. Non-detergent sodium carbonate solution was used to extract lipid microdomains [16]. Purified cellular plasma membrane was extracted with $2 \mathrm{ml}$ of $0.5 \mathrm{M} \mathrm{Na}_{2} \mathrm{CO}_{3}$ ( $\mathrm{pH} 11.0$ ), sonicated, and mixed with an equal volume of $90 \%$ sucrose, $50 \mathrm{mM}$ Mes and $0.3 \mathrm{M} \mathrm{NaCl}$ buffer ( $\mathrm{pH}$ 6.5). Four milliliters of extraction mixture was placed at the bottom of an ultra-centrifuge tube and overlaid with $4 \mathrm{ml}$ of $35 \%$ sucrose and $4 \mathrm{ml}$ of $5 \%$ sucrose in $25 \mathrm{mM} \mathrm{Mes}, 0.15 \mathrm{M} \mathrm{NaCl}$ and $0.25 \mathrm{M}$ $\mathrm{Na}_{2} \mathrm{CO}_{3}$. The gradient was formed in a $14 \times 89 \mathrm{~mm}$ Beckman centrifuge tube and centrifuged at $180000 \times g$ for $20 \mathrm{~h}$ in a SW41 rotor (Beckman) at $4{ }^{\circ} \mathrm{C}$. Fractions were collected from the top of the sucrose gradient.

A modified sucrose density gradient was developed to separate caveolae from other insoluble membrane lipid microdomains [15]. In this modified sucrose density gradient, $3 \mathrm{ml}$ of $35 \%$ sucrose solution, $4 \mathrm{ml}$ of $21 \%$ sucrose solution and $1 \mathrm{ml}$ of $5 \%$ sucrose solution were overlaid on $4 \mathrm{ml}$ of extraction mixture. The centrifugation conditions were not changed.

For detergent extraction $[17,18]$, the cell membrane was extracted with $2 \mathrm{ml}$ ice-cold Triton X-100 solution containing $0.5 \%$ Triton X-100, $25 \mathrm{mM}$ Mes (pH 7.0), 0.15 M NaCl, 5 mM EDTA, $1 \mathrm{mM}$ PMSF and $2 \mu \mathrm{l} / \mathrm{ml}$ protease inhibitors cocktail 1 and 2 [46] and mixed with an equal volume of $80 \%$ sucrose, $25 \mathrm{mM}$ Mes $(\mathrm{pH}$ 7.0), $0.15 \mathrm{M} \mathrm{NaCl}$ and $5 \mathrm{mM}$ EDTA. The sucrose gradient and centrifugation were the same as described in the previous section.

\section{Plasma membrane purification}

Confluent 3T3-L1 cells were washed with phosphate-buffered saline (PBS), scraped into isotonic buffer containing $250 \mathrm{mM}$ sucrose, $20 \mathrm{mM}$ Tris- $\mathrm{HCl}, 1 \mathrm{mM}$ EDTA, $15 \mathrm{mM} \mathrm{KCl,} 2 \mathrm{mM}$ $\mathrm{MgCl}_{2}, 1 \mathrm{mM} \mathrm{PMSF}$ and $2 \mu \mathrm{l} / \mathrm{ml}$ protease inhibitors cocktail 1 and 2 ( $\mathrm{pH}$ 7.5), and homogenized with a Dounce homogenizer. The cell lysate was centrifuged at $10000 \times \mathrm{g}$ for $45 \mathrm{~min}$ at $4{ }^{\circ} \mathrm{C}$, and the membrane pellet was resuspended in the same isotonic buffer. The plasma membrane was isolated by loading the resuspended membrane pellet onto a $1.12-\mathrm{M}$ sucrose cushion and centrifuging at $100000 \times g$ for $2 \mathrm{~h}$ [47]. The plasma membrane fraction at the interface was collected, diluted with PBS and pelleted by centrifugation at $200000 \times g$ for $60 \mathrm{~min}$.

\section{Western blot}

Samples of each fraction were subjected to SDS-PAGE and transferred to an Immobilon-P membrane (Millipore). The target proteins were revealed by western blot and enhanced chemiluminescence [5].

\section{Lipid analysis}

Cholesterol was extracted with 1.5 vol chloroform and vortexed for $4 \mathrm{~h}$ at $20^{\circ} \mathrm{C}$. After centrifugation, the organic phase was collected. The chloroform was evaporated and cholesterol was measured with the Infinity Cholesterol Reagents kit. The gangliosides (glycosphingolipids) were analyzed by dot blot with HRPconjugated cholera toxin $\beta$-subunit, as described by Hong et al. [5].
To determine the amount of other lipids, the sucrose in the isolated membrane lipid microdomains was diluted with PBS to less than $5 \%$. The membrane lipid microdomains were pelleted by centrifugation at $150000 \times g$ for $4 \mathrm{~h}$. All membrane proteins in the membrane lipid microdomains were recovered in the pellet as measured by the Lowry method and marker protein (caveolin-1 and aquaporin-1) western blot. The phospholipids and sphingomyelin were extracted with methanol and chloroform [28] and analyzed with thin-layer chromatography (Whatman, LK6D Silica gel) $[29,30]$.

\section{Electron microscopy}

Sucrose in the membrane samples was diluted with PBS. The membranes were pelleted by centrifugation at $150000 \times g$ for 4 h. The membrane pellets were fixed in $2.5 \%$ glutaraldehyde in PBS, washed with PBS and incubated in $1 \% \mathrm{OsO}_{4}$ in PBS. After gradual dehydration in ethanol, the fixed membranes were embedded in SPI-pon812 epoxy matrix (SPI Chem). The samples were observed by electron microscopy (Jeol JEM-1230).

\section{Proteomic analysis}

Lipid microdomains isolated by a sucrose density gradient were pelleted by centrifugation. The pellet was dissolved in $100 \mu \mathrm{l}$ reducing buffer containing $6 \mathrm{M}$ guanidine hydrochloride, $100 \mathrm{mM}$ ammonium bicarbonate and $10 \mathrm{mM}$ dithiothreitol ( $\mathrm{pH}$ 8.5). After incubation at $37^{\circ} \mathrm{C}$ for $30 \mathrm{~min}, 2.5 \mu \mathrm{l}$ of $1 \mathrm{M}$ iodoacetamide was added to the protein solution, which was incubated in the dark for an additional $30 \mathrm{~min}$ at room temperature. The sample was then buffer exchanged to $100 \mathrm{mM}$ ammonium bicarbonate ( $\mathrm{pH} 8.5)$, and digested with trypsin (1/50 amount of the protein) at $37^{\circ} \mathrm{C}$ overnight. One hundred micrograms of protein was used for each shotgun mass spectrometry analysis for protein identification [48, 49]. Briefly, the trypsin-digested peptides were applied to a ProteomeX Workstation (Thermo Finnigan, San Jose, CA, USA) for orthogonal 2D-LC-MS/MS analysis. The system was fitted with a strong cation exchange column (SCX, $320 \mu \mathrm{m}$, ID $\times 100 \mathrm{~mm}$, DEV SCX, Thermo Hypersil-Keystone) and two $\mathrm{C} 18$ reversed-phase columns (RP, $180 \mu \mathrm{m} \times 100 \mathrm{~mm}$, BioBasic ${ }^{\circledR} \mathrm{C} 18,5 \mu \mathrm{m}$, Thermo HypersilKeystone). The salt steps used were 0, 25, 50, 100, 200 and 800 $\mathrm{mM} \mathrm{NH}{ }_{4} \mathrm{Cl}$ synchronized with six $147-$ min reverse-phase gradients (from $100 \% \mathrm{~A}$ to $20 \% \mathrm{~A}$ and $80 \% \mathrm{~B}$ ). The reverse-phase solvents were $0.1 \%$ formic acid in water $(\mathrm{A})$ and $0.1 \%$ formic acid in acetonitrile (B). The effluent from the reverse-phase column was analyzed using a LCQ Deca XPplus ion-trap mass spectrometer. The micro-electrospray interface uses a $30-\mu \mathrm{m}$ metal needle that is orthogonal to the inlet of the LCQ. The mass spectrometer was set such that one full MS scan was followed by three MS/MS scans on the three most intense ions from the MS spectrum with Dynamic Exclusion $^{\mathrm{TM}}$. The acquired MS/MS spectra were automatically searched against protein databases using the TurboSEQUEST program in the BioWorks ${ }^{\mathrm{TM}} 3.1$ software suite. An acceptable SEQUEST result had to have a $\Delta C n$ score of at least 0.1 (regardless of charge state). Peptides with a +1 charge were accepted if they were fully tryptic-digested and had a cross-correlation $\left(X_{\text {corr }}\right)$ of at least 1.9. Peptides with a +2 charge were accepted if they had an $X_{\text {corr }} \geq 2.2$, and +3 charged peptides were accepted if they had an $X_{\text {corr }} \geq 3.75$. 


\section{Acknowledgments}

This work was supported by the National Natural Sciences Foundation of China (Grant nos: 90208007, 30521005 and 30623002) and the Ministry of Sciences and Technology of China (Grant nos: 2002CB513000 and 2006CB910700).

\section{References}

1 Rothberg KG, Heuser JE, Donzell WC, et al. Caveolin, a protein component of caveolae membrane coats. Cell 1992; 68:673-682.

2 Anderson RG. The caveolae membrane system. Annu Rev Biochem 1998; 67:199-225.

3 Drab M, Verkade P, Elger M, et al. Loss of caveolae, vascular dysfunction, and pulmonary defects in caveolin-1 genedisrupted mice. Science 2001; 293:2449-2452.

4 Razani B, Engelman JA, Wang XB, et al. Caveolin-1 null mice are viable but show evidence of hyperproliferative and vascular abnormalities. J Biol Chem 2001; 276:38121-38138.

5 Hong S, Huo H, Xu J, Liao K. Insulin-like growth factor-1 receptor signaling in 3T3-L1 adipocyte differentiation requires lipid rafts but not caveolae. Cell Death Differ 2004; 11:714723.

6 Simons K, Ikonen E. Functional rafts in cell membranes. $\mathrm{Na}$ ture 1997; 387:569-572.

7 Brown DA, London E. Function of lipid rafts in biological membranes. Annu Rev Cell Dev Biol 1998; 14:111-136.

8 Kurzchalia TV, Parton RG. Membrane microdomains and caveolae. Curr Opin Cell Biol 1999; 11:424-431.

9 Jacobson K, Mouritsen OG, Anderson RGW. Lipid rafts: at a crossroad between cell biology and physics. Nat Cell Biol 2007; 9:7-14.

10 Anderson RG, Jacobson K. A role for lipid shells in targeting proteins to caveolae, rafts, and other lipid domains. Science 2002; 296:1821-1825.

11 Mayor S, Rao M. Rafts: scale-dependent, active lipid organization at the cell surface. Traffic 2004; 5:231-240.

12 Lenne PF, Wawrezinieck L, Conchonaud F, et al. Dynamic molecular confinement in the plasma membrane by microdomains and the cytoskeleton meshwork. EMBO J 2006; 25:3245-3256.

13 Sharma P, Varma R, Sarasij RC, et al. Nanoscale organization of multiple GPI-anchored proteins in living cell membranes. Cell 2004; 116:577-589.

14 Gaus K, Gratton E, Kable EP, et al. Visualizing lipid structure and raft domains in living cells with two-photon microscopy. Proc Natl Acad Sci USA 2003; 100:15554-15559.

15 Yuan T, Hong S, Yao Y, Liao K. Glut-4 is translocated to both caveolae and non-caveolar lipid rafts but partially internalized through caveolae in insulin stimulated adipocytes. Cell Res 2007; 17:772-782.

16 Song KS, Li S, Okamoto T, Quilliam LA, Sargiacomo M, Lisanti MP. Co-purification and direct interaction of Ras with caveolin, an integral membrane protein of caveolae microdomains. J Biol Chem 1996; 271:9690-9697.

17 Deborah AB, Rose JK. Sorting of GPI-anchored proteins to glycolipid-enriched membrane subdomains during transport to the apical cell surface. Cell 1992; 68:533-544.

18 Sargiacomo M, Sudol M, Tang Z, Lisanti MP. Signal transducing molecules and glycosyl-phosphatidylinositol-linked proteins form a caveolin-rich insoluble complex in MDCK cells. $J$ Cell Biol 1993; 122:789-807.

19 Bickel PE, Scherer PE, Schnitzer JE, et al. Flotillin and epidermal surface antigen define a new family of caveolae-associated integral membrane proteins. J Biol Chem 1997; 272:1379313802.

20 Huo H, Guo X, Hong S, Jiang M, Liu X, Liao K. Lipid rafts/ caveolae are essential for insulin-like growth factor-1 receptor signaling during 3T3-L1 preadipocyte differentiation induction. J Biol Chem 2003; 278:11561-11569.

21 Drevot P, Langlet C, Guo XJ, et al. TCR signal initiation machinery is pre-assembled and activated in a subset of membrane rafts. EMBO J 2002; 21:1899-1908.

22 Causeret M, Taulet N, Comunale F, Favard C, Gauthier-Rouviere $\mathrm{C}$. N-cadherin association with lipid rafts regulates its dynamic assembly at cell-cell junctions in $\mathrm{C} 2 \mathrm{C} 12$ myoblasts. Mol Biol Cell 2005; 16:2168-2180.

23 Angst BD, Marcozzi C, Magee AI. The cadherin superfamily: diversity in form and function. J Cell Sci 2001; 114:629-641.

24 Schnitzer JE, Oh P. Aquaporin-1 in plasma membrane and caveolae provides mercury-sensitive water channels across lung endothelium. Am J Physiol 1996; 270:H416-H422.

25 Palestini P, Calvi C, Conforti E, et al. Compositional changes in lipid microdomains of air-blood barrier plasma membranes in pulmonary interstitial edema. J Appl Physiol 2003; 95:14461452.

26 Runembert I, Queffeulou G, Federici P, et al. Vimentin affects localization and activity of sodium-glucose cotransporter SGLT1 in membrane rafts. J Cell Sci 2002; 115:713-724.

27 Old WM, Meyer-Arendt K, Aveline-Wolf L, et al. Comparison of label-free methods for quantifying human proteins by shotgun proteomics. Mol Cell Proteomics 2005; 4:1487-1502.

28 Dodge JT, Phillips GB. Composition of phospholipids and of phospholipids fatty acids and aldehydes in human red cells. $J$ Lipid Res 1967; 8:667-675.

29 Wood WG, Cornwell M, Williamson LS. High performance thin-step chromatography and densitometry synaptic plasma membrane lipids. J Lipid Res 1989; 30:775-779.

30 Ruiz JI, Ochoa B. Quantification in the subnanomolar range of phospholipids and neutral lipids by monodimensional thinstep chromatography and image analysis. J Lipid Res 1997; 38:1482-1489.

31 Hancock JF. Lipid rafts: contentious only from simplistic standpoints. Nat Rev Mol Cell Biol 2006; 7:456-462.

32 Prior IA, Muncke C, Parton RG, Hancock JF. Direct visualization of Ras proteins in spatially distinct cell surface microdomains. J Cell Biol 2003; 160:165-170.

33 Schutz GJ, Kada G, Pastushenko VP, Schindler H. Properties of lipid microdomains in a muscle cell membrane visualized by single molecule microscopy. EMBO J 2000; 19:892-901.

34 Suzuki KG, Fujiwara TK, Sanematsu F, Iino R, Edidin M, Kusumi A. GPI-anchored receptor clusters transiently recruit Lyn and $\mathrm{G}$ for temporary cluster immobilization and Lyn activation: single-molecule tracking study 1. J Cell Biol 2007; 177:717-730

35 Suzuki KG, Fujiwara TK, Edidin M, Kusumi A. Dynamic 
recruitment of phospholipase $\mathrm{C}$ gamma at transiently immobilized GPI-anchored receptor clusters induces IP3-Ca2+ signaling: single-molecule tracking study 2. J Cell Biol 2007; 177:731-742.

36 Harding AS, Hancock JF. Using plasma membrane nanoclusters to build better signaling circuits. Trends Cell Biol 2008; 18:364-371.

37 Philippova MP, Bochkov VN, Stambolsky DV, Tkachuk VA, Resink TJ. T-cadherin and signal-transducing molecules co-localize in caveolin-rich membrane domains of vascular smooth muscle cells. FEBS Lett 1998; 429:207-210.

38 Doyle DD, Goings GE, Upshaw-Earley J, Pafe E, Ranscht B, Palfrey HC. T-cadherin is a major glycophosphoinositolanchored protein associated with noncaveolar detergent-insoluble domains of the cardiac sarcolemma. J Biol Chem 1998; 273:6937-6943.

39 Karlsson M, Thorn H, Danielsson A, et al. Colocalization of insulin receptor and insulin receptor substrate-1 to caveolae in primary human adipocytes. Cholesterol depletion blocks insulin signalling for metabolic and mitogenic control. Eur $J$ Biochem 2004; 271:2471-2479.

40 Parpal S, Karlsson M, Thorn H, Stralfors P. Cholesterol depletion disrupts caveolae and insulin receptor signaling for metabolic control via insulin receptor substrate-1, but not for mitogen-activated protein kinase control. J Biol Chem 2001; 276:9670-9678.

41 Muller G, Hanekop N, Wied S, Frick W. Cholesterol depletion blocks redistribution of lipid raft components and insulinmimetic signaling by glimepiride and phosphoinositolglycans in rat adipocytes. Mol Med 2002; 8:120-136.
42 Furuchi T, Anderson RGW. Cholesterol depletion of caveolae causes hyperactivation of extracellular signal-related kinase (ERK). J Biol Chem 1998; 273:21099-21104.

43 Pike LJ. Lipid rafts: bringing order to chaos. J Lipid Res 2003; 44:655-667.

44 Iwabuchi K, Handa K, Hakomori S. Separation of "glycosphingolipid signaling domain" from caveolin-containing membrane fraction in mouse melanoma B16 cells and its role in cell adhesion coupled with signaling. J Biol Chem 1998; 273:33766-33773.

45 Chigorno V, Palestini P, Sciannamblo M, et al. Evidence that ganglioside enriched domains are distinct from caveolae in MDCK II and human fibroblast cells in culture. Eur J Biochem 2000; 267:4187-4197.

46 Ronnett GV, Knutson VP, Kohanski RA, Simpson TL, Lane MD. Role of glycosylation in the processing of newly translated insulin proreceptor in 3T3-L1 adipocytes. $J$ Biol Chem 1984; 259:4566-4575.

47 Simpson IA, Yver DR, Hissin PJ, et al. Insulin-stimulated translocation of glucose transporters in the isolated rat adipose cells: characterization of subcellular fractions. Biochim Biophys Acta 1983; 763:393-407.

48 Washburn MP, Wolters D, Yates III RJ. Large-scale analysis of the yeast proteome by multidimensional protein identification technology. Nat Biotechnol 2001; 19:242-247.

49 Zhou H, Chen B, Li RX, et al. Large-scale identification of human biliary proteins from a cholesterol stone patient using a proteomic approach. Rapid Commun Mass Spectrom 2005; 19:3569-3578.

(Supplementary information is linked to the online version of the paper on the Cell Research website.) 